\title{
Körperliche Aktivität und Primärprävention
}

H.Löllgen, Remscheid, und Deborah Löllgen, Mainz

Sana-Klinikum Remscheid

\section{Physical activity and prevention of disease}

Physical inactivity is one of the major risk factors for cardiovascular diseases. Regular physical activity conversely is effective in reducing all-cause mortality, cardiovascular morbidity and mortality. Risks and side-effects can be neglected, medical examination before training is strongly recommended. Counseling healthy persons and patients at any age for physical activity, and training prescription should be an essential part of physicians' work in general practice.

Eingereicht: 17.3.2004 angenommen: 14.4.2004

\section{Einführung}

Herz-Kreislauferkrankungen stellen mit 41,3\% (Männer) bzw. 51,5\% (Frauen) auch in 2002 die häufigste Todesursache in Deutschland dar. Bewegungsmangel oder fehlende körperliche Aktivität liegt in über $80 \%$ aller Menschen über 30 Jahren vor. Diese Angaben sind vergleichbar für die europäischen Länder (Samitz $u$. Mensink (Hrsg.): Körperliche Aktivität in Prävention und Therapie, Hans Marseille Verlag, München 2002). Körperliche Inaktivität oder Bewegungsmangel sind seit längerem anerkannter Risikofaktor für kardiovaskuläre Erkrankungen (Am. Heart Ass., WHO). Der Wirkungsnachweis von Prävention durch körperliche Aktivität beruht einerseits auf Studien zu Immobilisation und Bewegungsmangel $(D$. K. McGuire et al.: Circulation 2001; 104: 1358-1369), andererseits auf trainingsphysiologischen Untersuchungen zur positiven Wirkung körperlicher Aktivität auf den Organismus. Epidemiologische Längsschnittstudien zur Prävention durch körperliche Aktivität erfolgten in den letzten 14 Jahren stets als prospektive Kohortenstudien. Doppel-blinde oder randomisierte Studien zu dieser Frage sind naturgemäß nicht möglich.

Unter körperlicher Aktivität versteht man alle muskulären Aktivitäten, die zu einer nachweisbaren Steigerung des Energieumsatzes führen. Sport ist hingegen eine muskuläre Beanspruchung mit Wettkampfcharakter oder mit dem Ziel einer persönlichen guten Leistung. Bewegung ist eine muskuläre Beanspruchung mit geringer Steigerung des Energieumsatzes (Samitz s.o., Hollmann, Hettinger: Sportmedizin, Schattauer-Verlag, Stuttgart/New York 2000, 4. Auflage).

\section{Trainingswirkungen}

Regelmäßige körperliche Aktivität hat vielfältige Auswirkungen auf den Organismus. Erste Anpassungen erfolgen stets in der arbeitenden Muskulatur. Muskelbioptisch lassen sich morphologische (z.B. Faserzusammensetzung) oder biochemische (Enzyme und Substrate) Veränderungen in der Muskelzelle nachweisen. An zweiter Stelle beobachtet man kardiovaskuläre Anpassungen mit einer Abnahme der Herzfrequenz und einer Zunahme der Herzfrequenzvariabilität als Hinweis auf die vagotone Lage. Es kommt zu einer verbesserten peripheren Ausschöpfung auf gleicher Belastungsstufe, zur Erhöhung der aerob-anaeroben Schwelle und einer Zunahme der maximalen Sauerstoffaufnahme und ventilatorischer Größen. Die funktionale pulmonale Alterung wird verlangsamt. Bei Bluthochdruck wird der arterielle Blutdruck gesenkt, Blutfettwerte und andere metabolische Größen werden günstig beeinflusst, thrombogene Faktoren gehemmt und gerinnungshemmende begünstigt. Das CRP als kardiovaskulärer Risikoindikator nimmt ab (Löllgen: Dtsch Arztebl 2003; 100: A 987-996).

\section{Epidemiologie}

Erste Metaanalysen (1990) zur Wirkung körperlicher Aktivität ergaben eine signifkante Senkung von Morbidität und Mortalität. Diese Studien waren noch inhomogen und nicht streng vergleichbar. Seitdem wurden über 30 weitere prospektive Kohortenstudien (Evidenzgrad IIa) in „peer reviewed“ Zeitschriften publiziert. In diesen Studien wurden über 400000 Personen erfasst mit einer Beobachtungsdauer bis zu 26 Jahren. Zusammengefasst ergaben diese Studien eine eindeutige Senkung der Gesamtmortalität (39\%) und der kardiovaskulären Mortalität (36\%) (Löllgen, s.o), auch bei Personen über 65 Jahren. Die Risikominderung durch Bewegung bleibt bestehen, wenn in den Studien die ersten 5 Jahre unberücksichtigt bleiben.

Viele dieser Untersuchungen zeigen eine Dosis-Wirkungsbeziehung, wobei leichtbis mittelgradige Belastungen einen günstigen Effekt haben, besonders hohe Trainingsintensitäten meist keinen zusätzlichen Effekt bringen. Bemerkenswert, dass kürzere tägliche Bewegungseinheiten (z.B. mehrfaches Treppensteigen) sich zu einer positiven Gesamtwirkung aufsummieren. Bewegung und körperliche Aktivität im Alltag haben eine protektive oder präventive Wirkung. Bedeutsam sind ebenfalls Befunde, wonach regelmässiges schnelles Spazierengehen einen Trainingseffekt und damit eine präventive Wirkung aufweist. Die Mortalität im Vergleich zu Inaktiven wird in Abhängigkeit von Gehstrecke und Geschwindigkeit gesenkt (Hakim: Circulation 1999; 100: 9-13). Auch bei Menschen über 65 Jahren ist eine kardiovaskuläre Prävention durch „Walking“ mit Senkung der Mortalität nachweisbar.

Leistungsfähigkeit und zukünftige Mortalität

In 13 Studien wurden die körperliche Leistungsfähigkeit mittels Ergometrie bestimmt und die Probanden im Längsschnitt beobachtet. Personen, die „fit“ 
Bewegung nehmen im Alter und bei hohem Trainingsumfang zu. Andererseits profitieren gerade ältere Menschen von einem umfassenden Trainingsprogramm mit Kraft- und Ausdauerkomponenten.

Trainingsempfehlungen

Mit mittlerem Trainings- und Übungsumfang werden ausreichende Trainingeffekte erzielt und präventiv wirksame Reize gesetzt. Optimal sind Trainingseinheiten von 30-45 Minuten 3 bis 4 mal pro Woche mit einer Trainingsintensität von etwa 50-70\% der maximalen Leistungsfähigkeit (DGSP-Empfehlung). Regelmäßige Belastungen im Alltag, auch längere Spaziergänge oder Wanderungen mit schnellem Tempo, sind eine sinnvolle und wirksame Ergänzung zum Training. Moderne Herzfrequenzmessgeräte unterstützen die Trainingssteuerung und helfen, Über- oder Unterbelastungen zu vermeiden. Neben dem Ausdauertraining (ca. 70\% des gesamten Trainingsumfangs) sind Kraft- und Beweglichkeitstraining (s.o., 30\% Umfang), vor allem im Alter, eine wichtige Ergänzung.

\section{Bewegung und Lebensstil}

Körperliche Aktivität wie auch Sport können kardiale Zwischenfälle bis hin zum Herztod begünstigen. Dies betrifft vor allem Personen, die neu, zu intensiv, nur sporadisch oder ohne sportärztliche Voruntersuchung mit dem Training beginnen. Mehrere Analysen zeigen, dass trotz eines geringen Risikos als Nettoeffekt körperliche Aktivität zu einer Senkung der kardialen Mortalität führt. Das Risiko wird gemindert, wenn eine Vorsorgeuntersuchung vor dem Trainingsaufbau erfolgt. Bei Personen über 35 Jahren mit mehr als einem Risikofaktor ist eine sportärztliche Untersuchung unbedingt zu empfehlen (Löllgen in: Samitz; DGSP-Empfehlungen: Leitlinie zur sportärztlichen Vorsorgeuntersuchung, 2002).

Risiken bestehen auch durch Verletzungen im Bereich des Bewegungsapparates. Ein ergänzendes Kraft- und Beweglichkeitsund Koordinationstraining senkt die Verletzungsgefahr, so wird bei Älteren die Sturzgefahr deutlich vermindert. Körperliches Training ist effektiver als Polsterungen zum Frakturschutz. Verletzungen durch chen Aktivität ist nicht die sportliche Leistung, sondern die vermehrte Bewegung und Aktivität im täglichen Leben. Bewegungen können an der Arbeitsstätte, zu Hause oder auch in öffentlichen Gebäuden in das Bewegungsprogramm eingebaut werden: Treppensteigen statt Aufzug, zu Fuß zum Briefkasten oder Einkaufen, regelmäßige häusliche Übungen zur Beweglichkeit und Kraftsteigerung. Anleitungen werden von den Sportvereinen („Sport pro Gesundheit") oder von qualifizierten Fitnessstudios angeboten. Jeder Mensch in jedem Alter sollte tägliche Bewegungseinheiten und mehrmalige Trainingseinheiten pro Woche in den Alltag integrieren. Der scheinbare Zeitverlust durch körperliche Aktivität wird durch konzentriertes Arbeiten und Leben in der übrigen Zeit ausgeglichen. Ziel der regelmäßigen körperlichen Aktivität ist nicht der Leistungssportler mit 75 Jahren, sondern der rüstige Mensch mit 80 oder 90 Jahren, der noch in der Lage ist, sich mit einer angemessenen Lebensqualität selbst versorgen zu können.
Körperliche Aktivität und Prävention anderer Erkrankungen

Körperliche Aktivität hat eine gesicherte präventive Wirkung bei Herz-Kreislaufund Gefäßerkrankungen. Bewegung senkt die Erkrankungshäufigkeit und Mortalität an Herzinfarkt, Schlaganfall und Bluthochdruck. Bei der arteriellen Verschlusskrankheit ist Gehen als Therapie allen anderen Verfahren überlegen. Körperliche Aktivität ist in der Lage, die zerebrale Funktion zu verbessern und den geistigen Abbau aufzuhalten (Hollmann, Strüder, Tagarakis: Nervenheilk 2003; 22: 467-474). Das wichtigste Behandlungsprinzip bei Osteoporose ist körperliche Aktivität mit Gehen und Laufen. Für drei Krebsarten stellt körperliche Aktivität eine gesicherte Prävention dar: Brustkrebs, Dickdarmkrebs und Prostatakrebs. Die Summe aller aufgeführten Effekte der körperlichen Aktivität verzögert nachweislich die biologische Alterung. Kein anderes Verfahren, keine Medikamente oder Heilweisen haben eine annähernd vergleichbare Verzögerung der Alterung aufzeigen können. Körperliche Aktivität hat auch bei der Sekundärprävention verschiedenster Erkrankungen, wie Herzinsuffizienz, Herzinfarkt und (noch zu selten) bei chronischen Lungenerkrankungen einen hohen und gesicherten Stellenwert.
Weitere Informationen: Deutsche Gesellschaft für Sportmedizin und Prävention (DGSP; www.dgsp.de).

Korrespondenz Prof. Dr. med. H. Löllgen Sana-Klinikum Remscheid

Burgerstr. 211

42859 Remscheid

DOI: $10.1055 / \mathrm{s}-2004-824858$

Fachliche Betreuung der
„Serie Prävention“:
Prof. Dr. Dr. h. c. Peter C. Scriba
Medizinische Klinik / Klinikum Innenstadt
der Universität München
Ziemssenstr. 1
80336 München
Prof. Dr. Friedrich W. Schwartz
Medizinische Hochschule Hannover
Abteilung für Epidemiologie, Sozialmedizin
und Gesundheitsforschung
Carl-Neuberg-Str. 1
30623 Hannover

